\title{
Direkte Demokratie und Parteienwettbewerb - Überlegungen zu einem obligatorischen Referendum als Blockadelöser auf Bundesebene
}

\author{
Eike-Christian Hornig
}

Volksabstimmungen erlangen eine immer größere Bedeutung in Europa und genießen viel Zuspruch bei den Bürgern, ganz im Gegensatz zu den Parteien. Auch in der Bundesrepublik wird ihre Einführung immer wieder als Reforminstrument auf Bundesebene gefordert, während sich auf der Landesebene direkte Demokratie inzwischen fest etabliert hat. Eine Übertragung dieser Konstellationen oder gar jener anderer Länder wie der Schweiz auf die Bundesebene ist allerdings aus verschiedenen Gründen kaum möglich. Ein entscheidender Grund dafür liegt darin, dass die Bundesrepublik im westeuropäischen Vergleich als ausgesprochener Parteienstaat gilt. ${ }^{1}$ In Anlehnung an das Party Government-Konzept von Richard Katz ${ }^{2}$ sprechen Heidrun Abromeit und Michael Stoiber von einer ausgeprägten Parteiendominanz. ${ }^{3}$ Diese äußert sich nicht nur in der Besetzung aller politischen Ämter durch die Parteien, sondern auch anhand der gesellschaftlichen Durchdringung von Interessenverbänden und zum Beispiel des öffentlich-rechtlichen Rundfunks. ${ }^{4}$ Die Implementierung „direkter Demokratie in den Parteienbundesstaat" ${ }^{\text {" } 5}$ bedarf daher besonderer Aufmerksamkeit. Ein zweiter wesentlicher Punkt ist dabei der deutsche Föderalismus. Die Abläufe und Eigenarten des Parteienwettbewerbes führen in Kombination mit dem Institutionensystem zu Mustern von Politik in der Bundesrepublik, die besonders für Reformen sehr hinderlich sein können. Ursächlich hierfür ist die Politikverflechtung im kooperativen Föderalismus mit einem de facto Zwangsverhandlungssystem, in dem die Entscheidungen der Bundesebene direkt von der Länderebene via Bundesrat abhängen - einstimmig oder nahezu einstimmig. ${ }^{6}$ Dabei ist die Versuchung zur parteitaktischen Blockade sehr groß. Dies hat unter den Stichworten Politikverflechtungsfalle $\mathrm{e}^{7}$ und Verhandlungsdemokratie ${ }^{8}$ Eingang in die Politik-

1 Vgl. stellvertretend Wilhelm Hennis, Auf dem Weg in den Parteienstaat. Aufsätze aus vier Jahrzehnten, Stuttgart 1998.

2 Vgl. Richard S. Katz, Party Government and its Alternatives, in: ders. (Hrsg.), Party Governments: European and American Experiences, Berlin / New York 1987, S. 1 - 26.

3 Vgl. Heidrun Abromeit / Michael Stoiber, Demokratien im Vergleich. Einführung in den Vergleich politischer Systeme, Wiesbaden 2006, S. 188.

4 Der Skandal um die Personalie des ZDF-Chefredakteurs Nikolas Brender ist hierfür ein Beispiel.

5 Frank Decker, Direkte Demokratie im deutschen „Parteienbundesstaat“, in: APuZ B10/2006, S. $3-10$.

6 Vgl. Fritz W. Scharpf, The Joint-Decision Trap: Lessons from German Federalism and European Integration, in: Public Administration, 66. Jg. (1988), H. 3, S. 239 - 278.

7 Vgl. Fritz W. Scharpf/ Bernd Reissert / Fritz Schnabel, Politikverflechtung. Theorie und Empirie des kooperativen Föderalismus in der Bundesrepublik, Kronberg 1976.

8 Vgl. Gerhard Lehmbruch, Parteienwettbewerb im Bundesstaat, Stuttgart 2000. 
wissenschaft gefunden. Die Möglichkeiten und Unmöglichkeiten von Reformen im Bundesstaat und des deutschen Föderalismus selber gehören dort zu den zentralen und vielbeachteten Themen, besonders nach zwei erfolgten und einer gescheiterten Reform. ${ }^{9}$ Der internationale Vergleich von verflochtenen Systemen lehrt dabei, dass besonders abgekoppelte Entscheidungs- und Verhandlungsarenen zum Erfolg von Reformen beitragen. ${ }^{10}$ In Bezug auf die Implementierung von direktdemokratischen Verfahren auf Bundesebene wäre also die erste Frage, inwiefern solche Verfahren generell mit diesen Anforderungen für erfolgreiche Verfassungsreformen korrespondieren. Zur Beantwortung wird in einem ersten Schritt anhand einer idealtypischen Unterscheidung verschiedener direktdemokratischer Instrumente ihr grundlegendes Reformpotential dargelegt. Aus diesen theoretischen Überlegungen folgt, dass direkte Demokratie prinzipiell Räume für abgekoppelte Entscheidungsund Verhandlungsarenen eröffnet, wenn auch mit Unterschieden je nach Instrument.

Die theoretische Annahme bei den abgekoppelten Arenen ist, dass sich Parteien neben anderen Akteuren wiederfinden, was ihr Verhalten zugunsten sachorientierter Motive verändert. Analytisch betrachtet bewegen sie sich im Prinzip zwischen drei alternativen Zielsetzungen. ${ }^{11}$ Das Streben nach Stimmen (,vote-seeking“) und Ämtern („office-seeking“) sind kurzfristig realisierbare Ziele, die auf die prozessuale Dimension von Politik gerichtet sind und insofern mit dem Begriff "politics" keinesfalls gleichgesetzt, doch aber in Verbindung gebracht werden können. Demgegenüber steht die Durchsetzung von bestimmten Inhalten („policy-seeking“), was nicht unbedingt mit dem schnellen Rhythmus repräsentativ-demokratischer Wahlen vereinbar sein muss. Hier wird die These vertreten, dass Blockaden unwahrscheinlicher werden, wenn Parteien langfristig und nicht taktisch-kurzfristige Motive verfolgen. Die zweite Frage ist also, inwiefern direktdemokratische Verfahren auch in der Praxis einen Handlungsraum darstellen, in dem Parteien weniger kurzfristige, taktische und mehr langfristige, inhaltliche Ziele verfolgen. Um sie zu beantworten, werden zunächst mögliche Motive von Parteien für die Auslösung von drei idealtypischen direktdemokratischen Verfahren herausgearbeitet. Mit diesem Schema werden dann die direktdemokratischen Praktiken auf nationaler Ebene anhand eines Originaldatensatzes von 141 Motiven von Parteien in neun westeuropäischen Ländern vergleichend analysiert. Dabei wird deutlich, dass besonders obligatorische Referenden aufgrund ihrer speziellen Konstruktionsweise weniger für eine parteitaktische, als für eine sachorientierte Nutzung geeignet sind. Dementsprechend lautet die dritte Frage, wie ein solches Verfahren auf Bundesebene in Deutschland aussehen könnte. Abschließend wird also ein Szenario eines möglichen obligatorischen Referendums entworfen, das den Implikationen entspricht. Dies steht im Einklang mit der Tendenz in der Literatur, die dieses Verfahren für am ehesten kompatibel mit den vorhandenen Gegebenheiten hält.

9 Vgl. Arthur Benz, Kein Ausweg aus der Politikverflechtung? Warum die Bundesstaatskommission scheiterte, aber nicht scheitern musste, in: PVS, 46. Jg. (2005), H. 2, S. 204 - 214; vgl. Frank Decker, In der Verflechtungsfalle. Möglichkeiten und Unmöglichkeiten einer Föderalismusreform, in: Bernhard Vogel / Rudolf Hrbek / Thomas Fischer (Hrsg.), Halbzeitbilanz, Baden-Baden 2006, S. $119-129$.

10 Vgl. Arthur Benz, Escaping Joint-Decision Traps: National and supranational experiences compared, in: Gerda Falkner (Hrsg.), Exits from the Joint-Decision Trap? Comparing EU Policies, Oxford 2011, S. 199 - 216.

11 Vgl. Kaare Strøm, A Behavioral Theory of Competitive Political Parties, in: American Journal of Political Science, 34. Jg. (1990), H. 2, S. $565-598$. 


\section{Förderliche Konditionen von Reformen}

Trotz einer Gegenwartsfixierung der Demokratie und der Anfälligkeit von Parteien für kurzfristige Lösungen befinden sich demokratische Systeme weltweit nicht im vollkommenen Stillstand. In den vergangenen Jahren hat eine Vielzahl von Verfassungsänderungen in den Demokratien Europas und Nordamerikas stattgefunden, darunter auch föderale Systeme mit ihren komplizierten Institutionengebilden, so zum Beispiel die Schweiz, Kanada oder eben die Bundesrepublik. Andere Länder wie Belgien, Spanien und Italien haben gar an ihren Grundfesten gerührt, indem sie ihre Verfassungen so verändert haben, dass sie sich in die Richtung von föderalen Systemen bewegen.

Arthur Benz hat in einem Vergleich von Reformen in nationalen und supranationalen Mehrebenensystemen vier förderliche Konditionen ausgemacht, um der Politikverflechtungsfalle zu entgehen. ${ }^{12}$ Nach den Arten der Konflikte und der Rigidität der jeweiligen Verfassungen zu urteilen, wäre in einem Fall wie Kanada von einem völligen Stillstand auszugehen, während Reformen in der Bundesrepublik als verhältnismäßig einfach erreichbar erscheinen. Das Gegenteil ist aber der Fall, denn in Kanada findet dennoch Wandel statt, der in Deutschland nur schwerfällig vorankommt. Es müssen also zusätzliche Faktoren für Reformen unter den Bedingungen von Politikverflechtung herangezogen werden.

Als erstes erwähnt Benz die Unterscheidung von regulärer Politik und Verfassungspolitik, die im Sinne einer Verlagerung von Prozessen auf die entsprechend andere Ebene neue Handlungsoptionen eröffnet. Der zweite Aspekt betrifft die Differenzierung von Handlungsarenen. So haben die Fälle Kanadas und der Schweiz gezeigt, dass eine Verlagerung in eigens eingerichtete Arenen mit Akteuren, die ansonsten nicht direkt in die intergouvernementalen Verhandlungsprozesse involviert sind, Möglichkeiten bietet, Verflechtungsfallen zu entgehen. So kann die Instrumentalisierung von Reformprojekten für kurzfristige, parteitaktische Ziele aufgeweicht und die Problemlösung forciert werden. Die Vergrößerung der Pluralität der Akteure führt den Verhandlungsprozess also in eine freiere Arena. Die dritte Möglichkeit ist die Sequenzialisierung von Verhandlungsprozessen durch die klare zeitliche Trennung der einzelnen Schritte. Dieser Logik folgt auch die vierte Möglichkeit: die Trennung von Verhandlungs- und Ratifikationsprozess. Die Chancen für ein Ergebnis erhöhen sich, wenn nicht dieselben Akteure beide Schritte des Ablaufes bestimmen. Allerdings beinhaltet die Möglichkeit der Volksabstimmung auch Ungewissheit, wie nach Benz die Fälle Kanada und Schweiz zeigen. Parteien sind also insgesamt an den Verfassungsreformprozessen beteiligt, aber das institutionelle „setting“ wird gegenüber dem regulären parlamentarischen Wege so verändert, dass sich auch die Verhandlungslogik wandelt.

Inwiefern direktdemokratische Verfahren diese Faktoren enthalten, hängt von ihrer institutionellen Gestaltung ab. Eine entsprechende Typologie stammt von Sabine Jung ${ }^{13}$, die wesentlich auf die Vorarbeiten von Silvano Möckli ${ }^{14}$ aufgebaut und seine sechs Kriterien durch die Aufhebung von logischen Überschneidungen auf vier reduziert hat: (1) Wer verfügt über die Kompetenz zur Auslösung einer Abstimmung? (2) Wer ist der Urheber der

12 Vgl. Arthur Benz, a.a.O. (Fn. 10).

13 Vgl. Sabine Jung, Die Logik direkter Demokratie, Wiesbaden 2001.

14 Vgl. Silvano Möckli, Nove democrazie a confronto, in: Mario Caciagli / Pier Vincenzo Uleri (Hrsg.), Democrazie e Referendum, Rom 1994. 


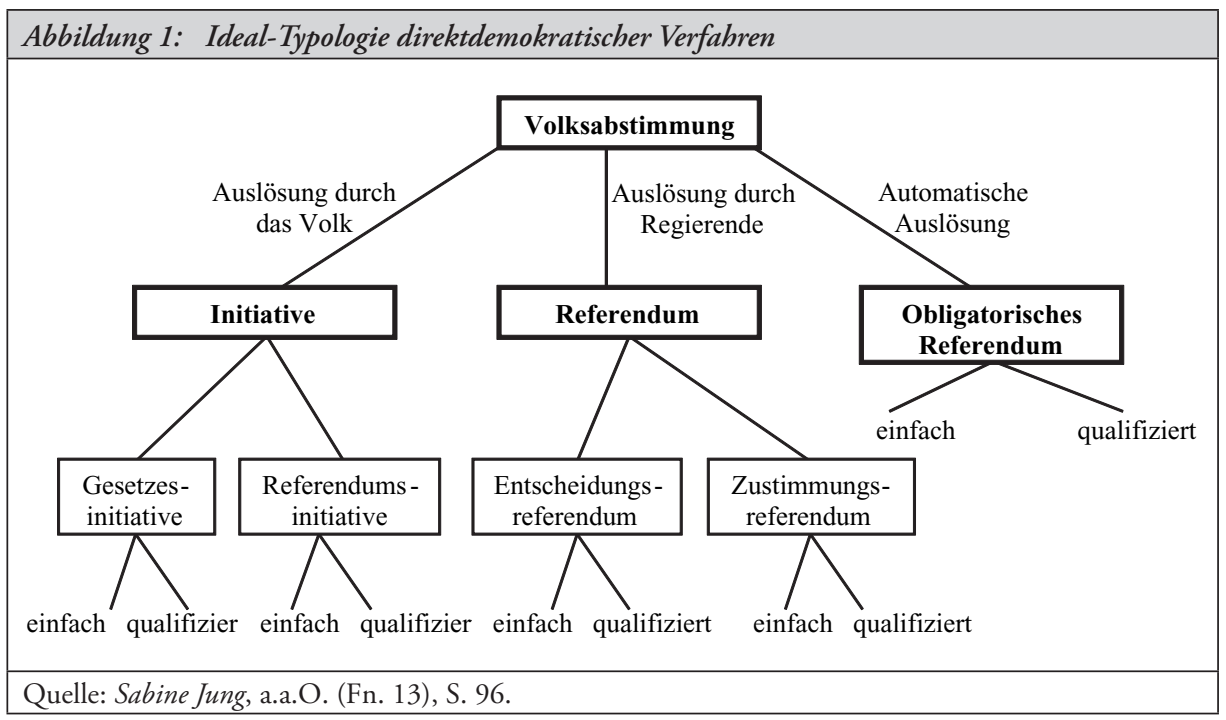

zur Abstimmung stehenden Vorlage? (3) Hat die Abstimmung Zustimmungs- oder Entscheidungscharakter? (4) Nach welchen Regeln wird entschieden? ${ }^{15}$

Demnach wird zwischen der Auslösung durch das Volk, durch Regierende und automatisch durch die Verfassung unterschieden. ${ }^{16}$ Die erste Variante entspricht dem Idealtyp der Initiative, die zweite dem des Referendums und die dritte dem des obligatorischen Referendums. Während die Urheberschaft beim Referendum genauso wie die Auslösung allein bei den Regierenden liegt, ist bei den offenen Verfahren zu unterscheiden: Bei Gesetzesinitiativen sind Auslösung und Urheberschaft offen, bei der Referendumsinitiative dagegen nur die Auslösung, während der Inhalt sich auf ein schon bestehendes Gesetz bezieht. Beim Referendum kann dagegen der Zeitpunkt der Entscheidung noch angeführt werden. Wird das Volk bei einem Referendum nachträglich befragt, handelt es sich um ein Zustimmungsreferendum. Entscheidet das Volk anstelle des Parlamentes, liegt ein Entscheidungsreferendum vor. Alle so definierten (durchgängigen) direktlegislativen Verfahren lassen sich noch hinsichtlich ihrer Entscheidungsregel, zum Beispiel nach Zustimmungs- oder Beteiligungsquoren, differenzieren.

Es wird klar, dass direkte Demokratie wesentliche Überschneidungen mit den Faktoren von Benz aufweist. Die Trennung von Aushandlungs- und Ratifikationsprozess durch die Entscheidung des Bürgers an der Urne ist logischer Bestandteil aller Verfahren. Alle drei Haupttypen können sich auf Verfassungspolitik beziehen, was von den jeweiligen nationalen Regelungen abhängt. Die Verfassungsinitiative in der Schweiz steht dabei beispielhaft für den ersten Idealtyp, das konstitutionelle Referendum in Italien für den zweiten, und das

15 Vgl. Sabine Jung, a.a.O. (Fn. 13), S. 81.

16 Die Bezeichnungen von Jung werden an dieser Stelle übernommen, obwohl sie zum Teil aufgrund ihrer mangelnden Präzision zu kritisieren sind. So wäre es sinnvoller, anstelle von Regierenden eher von Autoritären im Sinne von David Easton zu sprechen, da auch Oppositionsparteien oder subnationale Einheiten an Auslösungsprozessen beteiligt sein können. Zudem wäre eine Unterscheidung zwischen offenen und geschlossenen Verfahren hilfreich. 


\begin{tabular}{|c|c|c|c|c|}
\hline \multicolumn{5}{|c|}{$\begin{array}{l}\text { Tabelle 1: Übereinstimmung idealtypischer direktdemokratischer Verfahren mit den Faktoren für } \\
\text { Reformen in verflochtenen Systemen }\end{array}$} \\
\hline & Verfassungspolitik & Andere Arena & Sequenzialisierung & $\begin{array}{c}\text { Trennung } \\
\text { Verhandlung und } \\
\text { Ratifikation }\end{array}$ \\
\hline Initiative & (X) & $\mathrm{X}$ & $\mathrm{X}$ & $\mathrm{X}$ \\
\hline Referendum & $(\mathrm{X})$ & - & $(\mathrm{X})$ & $\mathrm{X}$ \\
\hline $\begin{array}{l}\text { Obligatorisches } \\
\text { Referendum }\end{array}$ & $\mathrm{X}$ & - & $(\mathrm{X})$ & $\mathrm{X}$ \\
\hline
\end{tabular}

obligatorische Referendum in Österreich exemplifiziert den dritten Idealtyp. Hauptsächlich sind Verfassungsfragen aber mit dem obligatorischen Mechanismus verbunden. Auch die Sequenzialisierung von Entscheidungsprozessen durch den Abstimmungsvorgang ist grundsätzlich gegeben, wobei dies allerdings durch den stufenweisen Prozess der Unterschriftensammlung und juristischen Überprüfung eher auf die Initiativen zutrifft. Besonders bei den offenen Verfahren der Volksgesetzgebung in den deutschen Bundesländern wird der Phasencharakter mit Volksbegehren und Volksentscheid deutlich. Beim Referendum und obligatorischen Referendum gibt es im Prinzip nur zwei Phasen: den parlamentarischen Entscheidungsprozess und die Abstimmung. Wenn die Sequenzialisierung der Herausnahme von Reformanläufen aus der normalen tagespolitischen Dynamik gilt, ist das hier also nur bedingt der Fall. Hinsichtlich der alternativen Handlungsarena kann nur die Initiative als eine solche gelten. Beim Referendum entscheiden allein die Parlaments- beziehungsweise Regierungsparteien über die Modalitäten des Verfahrens, beim obligatorischen Referendum zusammen mit der Verfassung. In beiden Fällen sind die Parteien in einer exklusiven Position. Die Initiative steht dagegen jedem Akteur offen, auch den Parteien. Sie brauchen nur genügend Unterschriften zu sammeln.

Die Initiative entspricht also fast allen Faktoren, die Benz für Wandel in verflochtenen Systemen als förderlich erachtet, so dass hier am ehesten mit Vorlagen zu rechnen ist, die außerhalb tagespolitischer Interessen der Parteien stehen. Das Referendum und das obligatorische Referendum unterscheidet dagegen nur die Frage nach der Verfassungspolitik, die beim Referendum nicht unbedingt dazu gehört. Charakteristisch ist außerdem, dass beide keine alternative Handlungsarena bereitstellen, da keine anderen Akteure als die im Parlament vertretenen Parteien beziehungsweise Regierungen beteiligt sind. Bei beiden Verfahren ist aufgrund dieser modellhaften Überlegungen ein deutlich höheres Maß an "politics“Nutzung durch die Parteien zu erwarten.

\section{Motive der Parteien bei der Auslösung von Volksabstimmungen}

Direktdemokratische Prozesse entsprechen nicht der klassischen Situation in einem Zwangsverhandlungssystem, in dem Parteien direkt miteinander verhandeln, sondern können ein Schachzug im Gesamtspiel sein, indem sie von Parteien in Ämtern ausgelöst werden. Es ist aber die Frage, ob die Auslösung einer Vorlage durch Parteien auf die parteipolitischen Wettbewerber zielt oder der Sache an sich dient. 
„Insbesondere wird - im Anschluss an Jürgen Habermas - der Direktdemokratie implizit unterstellt, dass ihre Ergebnisse sachpolitisch orientierter, ergebnisoffener, weniger von Interessen beeinflusst und bestenfalls in einem öffentlichen Diskurs entschieden werden. Ein solcher herrschaftsfreier, deliberativer Diskurs, in dem sich die einzelnen (,vernünftig agierenden') Partner als gleichwertig anerkennen, würde bedeuten, dass letztendlich die Kraft der Argumente entscheidet. ${ }^{17}$

Aus dieser deliberativen Sicht läge es nahe, den eher kurzfristigen parteipolitischen Interessen nur eine geringe Rolle zuzugestehen, da sie Partikularinteressen transportieren, die nicht unbedingt etwas mit der Richtigkeit eines Argumentes zu tun haben müssen. Wenn dessen Kraft überwiegt, somit Einsicht entscheidet, müssten alle Akteure dieselbe, argumentativ einzig sinnvolle Position vertreten. Die Frage nach taktischem, unsachlichem Einfluss der Parteien wäre obsolet, da die Wähler selbst vernünftig entschieden. Uwe Wagschal nennt diese Position die „Nullhypothese“, die Verbindungen zum Chancengleichheitspostulat der Pluralismustheorie aufweist. Neben diese „policy“-orientierte Perspektive lässt sich aber auch eine „politics“-Perspektive stellen:

„Die Gegenposition hierzu lässt sich auf Max Weber zurückführen, wonach Macht jede Chance bedeutet, innerhalb einer sozialen Beziehung den eigenen Willen auch gegen Widerstand durchzusetzen'. Volksabstimmungen sind von dieser Warte aus auch Machtfragen, in denen es um Ressourcen, Werte, Regelungen, Personen und andere politische Inhalte geht. Und politische Akteure, wie die Exekutive, Parteien und Verbände, haben dabei freilich auch ein vehementes Interesse, ihre Präferenzen, sprich: ihre ideologischen Ziele, gerade auch in direktdemokratischen Entscheidungen umzusetzen. "18

Dieser zweiten Sichtweise zufolge sind inhaltliche Ziele untrennbar mit Machtfragen verbunden, das heißt Abstimmungen bekommen auch über ihren Inhalt hinaus eine Bedeutung für die Parteipolitik. So kann zum Beispiel für eine Regierung die Ablehnung eines ihrer Gesetze das Ende bedeuten, auch wenn dies formal gar nicht zur Debatte steht. Oppositionsparteien werden demnach alles daran setzen, der schon wackelnden Regierung diese Niederlage beizufügen, selbst wenn sie der Vorlage inhaltlich aufgeschlossen gegenüberstehen. Beiden Positionen lassen sich konkrete Motive zuweisen, die allerdings von Verfahren zu Verfahren variieren.

Laurence Morel hat im Kontext der direktdemokratischen Praxis in Frankreich fünf Motive herausgearbeitet, die für die Auslösung von Abstimmungen durch Präsident beziehungsweise Regierung ausschlaggebend sind. Diese lassen sich auch auf andere direktdemokratische Kontexte und Parteien allgemein übertragen. Den ersten Umstand bezeichnet Morel als „mediation“:

„The mediation referendums have been organized to cope with a situation of internal divisions on a particular issue within the governing party and/or coalition. The referendum was supposed to circumvent the risk of a split, by allowing the government to escape the difficult task of imposing a policy on dissident sections of the party or the coalition. In

17 Uwe Wagschal, Diskurs oder Machtpolitik: Welche Interessen setzen sich in der Direktdemokratie am erfolgreichsten durch?, in: Markus Freitag / Uwe Wagschal (Hrsg.), Direkte Demokratie. Bestandsaufnahmen und Wirkungen im internationalen Vergleich, Münster 2007, S. 303 - 330, S. $303 \mathrm{f}$.

18 Ebenda, S. 304. 


\begin{tabular}{|l|l|l|c|}
\hline \multicolumn{2}{|c|}{ Tabelle 2: Übersicht der Motive der Parteien bei der Auslösung von Initiativen } \\
\hline Motiv & \multicolumn{1}{|c|}{ Umstände } & Art \\
\hline Agenda & Entscheidung vorholen oder aufschieben & & Politics \\
\cline { 1 - 2 } Legislativ & Oppositionsstatus ausgleichen & \multirow{2}{*}{ Oppositionsparteien } & Policy \\
\cline { 1 - 2 } Legitimation & $\begin{array}{l}\text { Rückhalt für eigene Politik / } \\
\text { Schwächung der Regierungsparteien }\end{array}$ & $\begin{array}{l}\text { Oppositionsparteien } \\
\text { mit einer Referendums- } \\
\text { initiative }\end{array}$ & Politics \\
\hline Veto & Beschlossenes Gesetz zu Fall bringen \\
\hline Quelle: Eigene Darstellung. & & \\
\hline
\end{tabular}

other words, the referendum was a means by which the government escaped responsibility for a specific decision, by transferring it to the people. "19

Die Delegation von Verantwortung spielt zweitens bei der Agendafunktion eine Rolle, wenn durch eine Abstimmung ein Thema aus Parlamentswahlen herausgehalten oder zumindest seine Wirkung minimiert werden soll. Bei Regierungsparteien ist dies besonders bei internen Spaltungen eine Option. Der dritte Aspekt betrifft die legislative Funktion eines Verfahrens, wenn andere Wege zu hohe Hürden aufweisen oder strukturell nicht erreichbar sind, wie etwa bei Minderheitsregierungen. Diese Funktion bezieht sich innerhalb der Gruppe der Initiativen allerdings nur auf die Gesetzesinitiative, da es bei Referendumsinitiativen primär um die Verhinderung bereits beschlossener Gesetze geht. Viertens dienen die von Regierungen angesetzten Volksabstimmungen nicht nur der Gesetzgebungs-, sondern auch der Legitimationsfunktion. Die primär personengebundene Nutzung von Volksabstimmungen, fünftens, bezeichnet Laurence Morel besonders als französisches Phänomen, was angesichts der Instrumentalisierung der direkten Demokratie durch Präsident Charles de Gaulle keine Überraschung ist. ${ }^{20}$ In Ergänzung zu Morel ist noch die Vetofunktion zu nennen. Oppositionsparteien können im Parlament eine Abstimmung über ein von der Mehrheit beschlossenes Gesetz auslösen. Die Regierungspolitik würde zugleich durch die Androhung eines Referendums beeinflussbar werden. Insgesamt ergeben sich also fünf Motive für Parteien eine Volksabstimmung zu betreiben: die Mediations-, die Agenda-, die Gesetzgebungs-, die Legitimations- und die Vetofunktion. Diese korrespondieren unterschiedlich mit kurzfristig parteipolitischen oder eher langfristig inhaltlichen Zielen, wie die folgenden Erörterungen getrennt nach Verfahren zeigen. ${ }^{21}$

19 Laurence Morel, The Rise of 'Politically Obligatory' Referendums: The 2005 French Referendum in Comparative Perspective, in: West European Politics, 30. Jg. (2007), H. 5, S. 1041 - 1067, S. 1045.

20 Ebenda, S. 1050.

21 Die Erwägungen über Initiativen betreffen nur Parteien der Opposition, da nur diese im Regelfall ein Motiv für die Auslösung einer Initiative beziehungsweise Referendumsinitiative haben. Dass Regierungsparteien per Unterschriftensammlung ein Gesetz schaffen oder es mit einem Veto stoppen wollen, ist genauso unlogisch, wie dass eine zerstrittene Mehrheitspartei eine Gesetzesinitiative betreibt, um ihre internen Probleme zu lösen. Daher fallen Regierungsparteien aus der Betrachtung heraus. 
Nicht alle von Morel unterschiedenen Motive sind bei offenen Verfahren sinnvoll, so etwa die Mediation. Es wird keine Oppositionspartei eine Unterschriftensammlung für eine Gesetzesinitiative betreiben, um einer Zerreißprobe zu entgehen. Viel eher würde die Entscheidung vertagt, da in der Opposition gar keine Notwendigkeit zur Entscheidung besteht. Auch die personengebundene Nutzung fällt weg, da sie das logische Gegenteil der offenen Auslösung ist. Übrig bleiben vier Motive (der Oppositionsparteien) für die Auslösung einer Gesetzes- oder Referendumsinitiative.

Die Agenda- und die Legitimationsfunktion haben einen deutlichen „politics“-Charakter. Erstere entspringt der Notwendigkeit, eine direktdemokratische Entscheidung aufschieben oder vorziehen zu müssen, um parteipolitisch ungünstige Konstellationen abzuwenden. Klassisches Beispiel ist eine anstehende Wahl, aus der ein parteipolitisch ungünstiges Thema durch eine vorher abgehaltene Entscheidung herausgehalten werden soll. Eine Wahl könnte auch bei der Legitimationsfunktion eine Rolle spielen, wenn eine direktdemokratische Initiative besonders zur Profilierung im Vorfeld von einer Partei genutzt wird. Die Gesetzgebungs- und die Vetofunktion entsprechen dagegen der „policy“-Orientierung. Zwar können Parteien eine Gesetzesinitiative betreiben, um die Regierungsarbeit zu stören, doch wird dieser Umstand schon mit der Legitimationsfunktion abgedeckt. Bei der legislativen Funktion geht es primär um den Versuch der Durchsetzung von Inhalten. Die Vetofunktion ergibt sich nur im Zusammenhang einer Referendumsinitiative, da Gesetzesinitiativen einen neuen Gesetzesvorschlag transportieren und nicht als Veto fungieren können. Dabei kann schon die Androhung einer Referendumsinitiative eine Vetofunktion entfalten, ohne dass es tatsächlich zur Abstimmung kommen muss.

Bei Referenden sind aufgrund der geschlossenen Auslösung eigentlich nur die Motive der Regierungspartei(en) zu berücksichtigen. Oppositionsparteien können lediglich relevant werden, wenn etwa zur Auslösung eines Referendums eine qualifizierte Mehrheit im Parlament notwendig ist. Dabei ergeben sich fünf Motive für Parteien, ein Referendum zu betreiben. Die Mediationsfunktion steht im Zeichen der Behebung oder Minderung einer

\begin{tabular}{|l|l|l|c|}
\hline \multicolumn{1}{|c|}{ Tabelle 3: Auslösung von Referenden durch Parteien } & Art \\
\hline Motiv & \multicolumn{1}{|c|}{ Ziel } & \multicolumn{1}{|c|}{ Umstände } & Politics \\
\hline Mediation & $\begin{array}{l}\text { Innere Spaltung } \\
\text { vermeiden }\end{array}$ & $\begin{array}{l}\text { Kontroverse in Mehrheitspartei } \\
\text { (-koalition) }\end{array}$ & Politics \\
\hline Agenda & $\begin{array}{l}\text { Entscheidung vorholen } \\
\text { oder aufschieben }\end{array}$ & $\begin{array}{l}\text { Handlungsdruck von Außen / Position } \\
\text { der Schwäche; Günstige Gelegenheit / } \\
\text { Position der Stärke }\end{array}$ & Policy \\
\hline Legislativ & $\begin{array}{l}\text { Widerstände umgehen } \\
\text { (Druck von Oppositions- } \\
\text { parteien) }\end{array}$ & $\begin{array}{l}\text { Minderheitsregierung; Supermajoritäten } \\
\text { erforderlich }\end{array}$ & Policy \\
\hline Legitimation 2 & $\begin{array}{l}\text { Reülärkhalt für eigene Politik / } \\
\text { on / Zufriedenstellung } \\
\text { wichtiger Klientelgruppen }\end{array}$ & $\begin{array}{l}\text { Position der Stärke; Position der Schwäche } \\
\text { der Schwäche (Druck von Außen) }\end{array}$ & Politics \\
\hline Veto & $\begin{array}{l}\text { Inkrafttreten eines Gesetzes } \\
\text { verhindern }\end{array}$ & Auslösung durch Oppositionsparteien & Policy \\
\hline Quelle: Eigene Darstellung. & \multicolumn{2}{l}{} \\
\hline
\end{tabular}


parteiinternen Spaltung durch die Auslagerung der Entscheidung. Bei der Agendafunktion wird eine direktdemokratische Entscheidung auf einen für die beteiligte Partei günstigeren Termin verschoben oder vorgezogen. Dies kann aus einer Position der Schwäche und Stärke heraus passieren. Bei der legislativen Funktion steht dagegen wieder die „policy“-Prägung im Vordergrund. Es geht primär um die Umsetzung von Inhalten. Die Legitimationsfunktion dagegen kann beide Charakterisierungen annehmen. Die Legitimation eines Gesetzesvorhabens entspricht der „policy“-Dimension. Ziel ist die Herstellung eines breiten gesellschaftlichen Konsenses über ein Thema. Die Stärkung der Legitimation einer Partei, wie bei der zweiten Variante, entspricht der „politics“-Dimension. Hier ist es egal, ob der Hintergrund eine Position der Schwäche oder Stärke der Partei ist, da die Auslösung der Abstimmung im Kontext des parteipolitischen beziehungsweise parlamentarischen Wettstreites steht. Die Vetofunktion entspricht wieder der „policy“-Dimension.

Obligatorische Referenden stehen im Kontext von Verfassungsänderungen oder Übertragungen von Souveränität. Aus der Bedeutung und der „Schwere“ der betreffenden Materie erwächst der gesetzliche Zwang zur Abstimmung bei einer Veränderung des legislativen Status Quo. Es wäre daher naheliegend, bei obligatorischen Referenden keine relevanten Umstände der Auslösung zu vermuten. Demnach würden alle Abstimmungen eine Legitimationsfunktion erfüllen und Parteien allein auf die Urheberschaft Einfluss nehmen können. Doch auch bei der Auslösung von obligatorischen Referenden kann nach dem Kalkül von Parteien gefragt werden. Beispielsweise kann eine Verfassungsänderung vorgezogen werden, um ein Thema aus dem anstehenden Wahlkampf herauszuhalten. Denkbar ist auch, dass Verfassungsänderungen aufgrund von Massendemonstrationen erfolgen. Allerdings kann auch ein breiter gesellschaftlicher Konsens zur Änderung eines bestimmten Gesetzes bestehen, so dass die tatsächliche Legitimationsfunktion des Verfahrens im Vordergrund steht. Beispiele für derartige obligatorische Referenden lassen sich neben der Schweiz und Österreich auch in Dänemark finden. ${ }^{22}$

In Frage kommen demnach die Agenda-, die Legitimations- und die Vetofunktion. Weggelassen werden die Mediation und die personengebundene Nutzung. In der Regel werden bei obligatorischen Volksabstimmungen Entscheidungen des Parlamentes dem Wähler zur Ratifizierung vorgelegt, so dass diese nur schwerlich eine Ausflucht aus einer inneren Spaltung sein können, da es zuvor im Parlament galt, Farbe zu bekennen. Das personengebundene Motiv liegt quer zur Verfahrensgestaltung und -nutzung. Die legislative Funktion fällt ebenfalls weg, da die Parlamentsmehrheit oder Minderheitsregierung nicht versuchen kann, über den Umweg einer Abstimmung eine Blockade zu umgehen.

Es zeigt sich im Vergleich der Verfahren, dass ihre Auslösung durch Parteien sowohl aus parteitaktischen, eher kurzfristigen „politics“-Überlegungen als auch aus eher inhaltlich bestimmten, über den Tag der nächsten Wahl hinausgehenden „policy“-Erwägungen heraus erfolgen kann.

22 Zudem können auch teilobligatorische Verfahren ausgemacht werden, bei denen beim Unterschreiten einer bestimmten qualifizierten Mehrheit im parlamentarischen Prozess eine Abstimmung automatisch ausgelöst wird. Ist dieses Zustimmungsquorum erfüllt, entfällt auch der automatische Zwang zur Abstimmung. In einem solchen Fall kann die Ablehnung einer Verfassungsänderung durch eine kleine Partei schon den Ausschlag zugunsten einer Abstimmung geben. Das Referendum bei der Übertragung von Souveränität in Dänemark wäre hierfür ein Beispiel. Es reicht die Ablehnung durch ein Sechstel der Parlamentsabgeordneten für eine Abstimmung. 


\begin{tabular}{|l|l|l|c|}
\hline Tabelle 4: Auslösung von obligatorischen Referenden & \multicolumn{1}{|c|}{ Umstände } & Art \\
\hline Motiv & \multicolumn{1}{|c|}{ Ziel } & $\begin{array}{l}\text { Handlungsdruck von Außen / Position } \\
\text { der Schwäche; Günstige Gelegenheit / } \\
\text { Position der Stärke }\end{array}$ & Politics \\
\hline Agenda & $\begin{array}{l}\text { Entscheidung vorholen } \\
\text { oder aufschieben }\end{array}$ & Kaum parteipolitischer Streit & Policy \\
\hline Legitimation 1 & Gesellschaftlicher Konsens & Politics \\
\hline Legitimation 2 & $\begin{array}{l}\text { Rückhalt für eigene Politik / } \\
\text { Position }\end{array}$ & $\begin{array}{l}\text { Ohne Supermajoritäten / } \\
\text { Position der Stärke }\end{array}$ & Policy \\
\hline Veto & $\begin{array}{l}\text { Inkrafttreten eines Gesetzes } \\
\text { verhindern }\end{array}$ & $\begin{array}{l}\text { Auslösung bei Unterschreitung von } \\
\text { Supermajoritäten im Vorfeld }\end{array}$ \\
\hline Quelle: Eigene Darstellung. & & \\
\hline
\end{tabular}

\section{Parteimotive im westeuropäischen Vergleich}

Die Grundlage der Analyse ${ }^{23}$ ist ein Originaldatensatz mit 141 Motiven von Parteien bei 131 Abstimmungen in Italien, Großbritannien, Norwegen, Dänemark, Schweden, den Niederlanden, Österreich, Frankreich ${ }^{24}$ und der Schweiz. Zugrunde gelegt wurden alle Abstimmungen seit 1945 und in der Schweiz seit 1999. Damit wurden sowohl offene (Schweiz und Italien) als auch geschlossene Verfahren (in den restlichen Ländern), sowohl häufig (Schweiz und Italien) als auch selten oder einmalig genutzte Verfahren (Niederlande und Großbritannien) berücksichtigt. Bei der Initiative werden dabei nur jene Abstimmungen mit einbezogen, an deren Auslösung auch Parteien beteiligt waren. Hinzu kommen national sehr differenzierte direktdemokratische Institutionen (Dänemark mit sechs unterschiedlichen Verfahren; Italien und die Schweiz mit jeweils drei) und national auf einen Mechanismus beschränkte Erfahrungen (Großbritannien, Niederlande und Norwegen). Die erfassten Motive der Parteien werden hier erstmals einzeln für jeden Verfahrenstyp dargestellt.

Das Ergebnis der empirischen Untersuchung bestätigt grundsätzlich die theoretischen Annahmen, wenn auch mit deutlichen Unterschieden zwischen den Verfahren. Der internationale Vergleich zeigt direkte Demokratie als grundsätzlich geeigneten Mechanismus für Reformen. Die Auslösung von Abstimmungen durch Parteien wird mehrheitlich durch „policy“-orientierte Motive verursacht (58,5 Prozent). Dem stehen 41,4 Prozent der ermittelten Motive gegenüber, bei denen kurzfristige, parteitaktische Erwägungen für die Auslösung einer Abstimmung ursächlich waren. In diesen Fällen wird direkte Demokratie von den Parteien instrumentalisiert, um sich insbesondere Vorteile bei anstehenden Wahlen

23 Die Ausführungen und Daten basieren auf Eike-Christian Hornig, Die Parteiendominanz direkter Demokratie in Westeuropa, Baden-Baden 2011. Dafür wurden für jedes Land mindestens alle Parlamentsparteien beziehungsweise auch Parteien mit mehr als zwei Prozent der gültigen Stimmen der letzten Parlamentswahl herangezogen. Jedes einzelne Parteimotiv wurde aus dem jeweiligen Kontext heraus qualitativ den oben unterschiedenen Motiven zugeordnet, so dass sich additiv ein Gesamtbild ergibt, wie es in Tabelle 5 zu sehen ist.

24 Aus der französischen direktdemokratischen Praxis werden nur die beiden Verfassungsreferenden aus den Jahren 1958 und 2000 berücksichtigt, da das Präsidentielle Referendum nach Artikel 11 der Verfassung im vorliegenden Zusammenhang einer möglichen Übertragbarkeit der Ergebnisse aufgrund seiner speziellen Konstruktion im Kontext des semi-präsidentiellen Systems nicht in Frage kommt. 
$\mathrm{zu}$ verschaffen. $\mathrm{Zu}$ beachten sind allerdings wiederum die Unterschiede zwischen den Verfahren.

Die Nutzung von Initiativen durch Parteien ist im westeuropäischen Vergleich mehrheitlich „policy“-orientiert gewesen und bestätigt damit die theoretischen Implikationen der Faktoren von Benz, wenn auch nicht so deutlich. Das Verhältnis gegenüber der „politics“-Dimension ist 59 zu 41 Prozent. Das dominierende Motiv für die Auslösung durch Parteien ist die legislative Funktion. In 37 Prozent der Fälle veranlasste die Unfähigkeit, auf einem anderen Wege einen neuen legislativen Status Quo in einem bestimmten Bereich herbeizuführen, Parteien dazu, eine Initiative zu betreiben. Dies weist auf die Bedeutung von offenen Verfahren insbesondere für Parteien in der Opposition hin. Etwa 22 Prozent aller betriebenen Initiativen dienten einer Vetofunktion. Dies waren Referendumsinitiativen. In mehr als einem Drittel der Fälle spielte die parteitaktisch motivierte Legitimationsfunktion die ausschlaggebende Rolle. Es ging darum, Rückhalt für die Politik beziehungsweise Position der entsprechenden Partei zu generieren oder die Regierungsparteien zu schwächen. Offene Verfahren sind besonders für kleine Parteien eine Möglichkeit, Aufmerksamkeit auf sich zu lenken und sich zu profilieren. ${ }^{25}$ Dies ist bei der Analyse der Verfassungsinitiative in der Schweiz deutlich geworden, wo vor Wahlen zum Beispiel die Schweizer Demokraten, aber auch die Sozialdemokraten mit Vorlagen entsprechend in Erscheinung getreten sind. Eine geringe Rolle spielte mit nur sechs Prozent der Motive die Agendafunktion. Die Erfahrungen aus der Schweiz und Italien zeigen, dass Initiativen durchaus als Verfahren für große Reformprojekte in Frage kommen. ${ }^{26}$ Allerdings spricht die offene Auslösung und Urheberschaft auch für eine mögliche Unbeständigkeit im Nutzungsverhalten, das heißt je nach politischen Konstellationen kann sich das Verhältnis von „politics“- und „policy“-Nutzung wieder verändern.

Bei den beiden geschlossenen Verfahren ergeben sich dagegen deutliche Unterschiede in den Ergebnissen. Im Vordergrund steht das obligatorische Referendum, das fast vollständig im Zeichen „policy“-orientierter Politik genutzt wurde (92 Prozent). Die entsprechende

\begin{tabular}{|l|c|c|c|}
\hline Tabelle 5: Motive getrennt nach Verfahrenstyp (in Prozent) \\
\hline & Initiative & Referendum & $\begin{array}{c}\text { Obligatorisches } \\
\text { Referendum }\end{array}$ \\
\hline "politics“-orientierte Motive & & & \\
Mediation & 0 & 17 & 0 \\
Agenda & 6 & 17 & 4 \\
Legitimation & 35 & 26 & 4 \\
Total & 41 & 60 & 8 \\
\hline "policy“-orientierte Motive & 0 & & 59 \\
Legitimation & 37 & 17 & 4 \\
Legislativ & 22 & 20 & 29 \\
Veto & 59 & 40 & 92 \\
Total & & \\
\hline Quelle: Eigene Darstellung. & \multicolumn{3}{|}{} \\
\hline
\end{tabular}

25 Vgl. Lutz Hager, Wie demokratisch ist direkte Demokratie?, Wiesbaden 2005.

26 Vgl. zum Beispiel Anna Capretti, Die Öffnung der Machtstrukturen durch Referenden in Italien, Frankfurt am Main 2001. 
Legitimationsfunktion dominiert unter den ermittelten Motiven mit einem Anteil von 59 Prozent. In diesen Fällen diente direkte Demokratie tatsächlich der Herstellung eines breiten gesellschaftlichen Konsenses bei einer Entscheidung mit besonderer Tragweite. Hinzu kommen 29 Prozent Vetomotive, wenn Parteien ihre Sperrminorität ausnutzten, um eine Abstimmung auszulösen und ein bestimmtes Gesetz aufzuhalten. Aber auch Regierungsparteien votierten für Abstimmungen, wenn sie eine parlamentarische Blockade oder ein Patt innerhalb der Regierungskoalition umgehen wollten. Das obligatorische Referendum kommt damit der „Nullhypothese“ der direkten Demokratie von Wagschal nahe. ${ }^{27}$ Nur acht Prozent der Motive hatten einen parteitaktischen Hintergrund (Agenda- und Legitimationsfunktion).

Referenden schließlich sind am deutlichsten von der "politics“-orientierten Nutzung durch Parteien geprägt. Hier schlägt der Antagonismus von Regierung und Opposition und damit von rivalisierenden Parteien beziehungsweise Parteienkoalitionen durch. In 60 Prozent der Fälle sind Referenden aus den Mechanismen, Logiken und Dynamiken des taktischen Parteienwettbewerbes heraus entstanden. Am häufigsten (26 Prozent) dienten sie den Parteien zur zusätzlichen Legitimation im parteipolitischen Wettstreit. Regierungsparteien haben es auch häufig auf die Mediationsfunktion von Abstimmungen abgesehen (17 Prozent), um eine Zerrreißprobe bei einem parteiintern umstrittenen Thema zu vermeiden. In diesem Zusammenhang steht auch die Agendafunktion mit ebenfalls 17 Prozent der Motive. Druck von außen, zum Beispiel durch Parlamentswahlen, zwingt die Parteien zum Handeln. Regierungsparteien versuchen, für sie ungünstige Themen durch Abstimmungen aus Wahlkämpfen herauszuhalten. Besonders die Sozialdemokraten in Skandinavien hatten ihre liebe Mühe und Not mit den Abstimmungen im Kontext der europäischen Integration, da sie in der Bevölkerung meistens spaltend wirkten. ${ }^{28}$ Der effektivste Weg, wie die Parteien ungeliebte Themen gleichsam in Quarantäne stecken konnten, war, diese zeitlich vom regulären Parteienwettbewerb zu isolieren.

Die drei „policy“-orientierten Motive kommen zusammen auf 40 Prozent, wovon die Hälfte auf die Vetofunktion zurückgeht, was erklärungsbedürftig ist, da Referenden geschlossene "top-down“-Verfahren sind. Doch optieren häufig Parteien der Opposition für Abstimmungen, um Projekte der Regierungsmehrheit zu stoppen. Wenn Verfahren als Instrumente zum Minderheitenschutz konzipiert sind, wie in Dänemark das fakultative $\mathrm{Zu}$ stimmungsreferendum, dann kann auch die Minderheit Abstimmungen auslösen. Gegenüber dem Motiv Legitimation 1 mit einem Anteil von 17 Prozent spielte die legislative Funktion mit nur einem Anteil von drei Prozent keine Rolle. Hintergrund sind hier die Versuche von einzelnen Parteien, zum Beispiel den italienischen Sozialisten im Jahr 1987, aus ihren Koalitionsregierungen auszuscheren und Inhalte auch gegen die eigentlichen Koalitionspartner in einer neuen referendumsbezogenen Koalition durchzusetzen. Dass dies nicht unnötig auf Kosten der Bündnispartner gehen sollte (wie es der Legitimationsfunktion entsprechen würde), ergibt sich aber aus der Koalitionssituation, an der dennoch festgehalten werden sollte. ${ }^{29}$

27 Vgl. Uwe Wagschal, a.a.O. (Fn. 17), S. 303.

28 Vgl. Nicolas Aylott, Lessons Learned, Lessons Forgotten: The Swedish Referendum on EMU in September 2003, in: Government and Opposition, 40. Jg. (2005), H. 4, S. 540 - 564, S. 541.

29 Vgl. Augusto Barbera / Andrea Morrone, La repubblica dei referendum, Bologna 2003, S. 95 f. 
Der Unterschied zwischen den Motivlagen der Parteien bei der Nutzung von Referenden und obligatorischen Referenden führt zu grundlegenden Schlussfolgerungen: Erstens können die vier Faktoren von Arthur Benz hier nicht den ausschlaggebenden Unterschied machen, da beide Verfahrenstypen fast identisch drei von vier Kriterien entsprechen. Das obligatorische Referendum weist eine höhere „policy“-Quote auf, stellt aber keine alternative Handlungsarena im Sinne von zusätzlichen Akteuren dar. Dies unterstreicht zweitens die Bedeutung der institutionellen Unterschiede der Verfahren für die Art der Nutzung durch die Parteien. Als wesentlicher Punkt schält sich hier die Kompetenz zur Auslösung heraus, die die Parteien beim obligatorischen Referendum mit der Verfassung teilen müssen. Dort wo sie darüber verfügen, nämlich bei normalen Referenden, beläuft sich der Anteil der parteitaktischen Motive, bei denen die Kompetenz zur Auslösung der Schlüssel ist, sogleich auf ein Drittel (Agenda und Mediation). Dass die Parteien die Initiativen trotz ihrer ebenfalls offenen Auslösung und Urheberschaft nicht auch noch deutlicher parteitaktisch für sich nutzen, liegt an der Sinnlosigkeit der Motive Mediation und Agenda in diesem Zusammenhang, da Initiativen fast ausschließlich von Oppositionsparteien genutzt werden.

\section{Entwurf eines obligatorischen Referendums bei Verfassungsänderungen auf Bundesebene in Deutschland}

In der Bundesrepublik hat auf der Landesebene bereits ein „regelrechter Siegeszug der Plebiszite“ ${ }^{30}$ stattgefunden. In allen deutschen Bundesländern fungieren direktdemokratische Verfahren inzwischen als Mechanismen der Entscheidungsfindung ergänzend zu den repräsentativen Institutionen. ${ }^{31}$ Zwar konzentriert sich die Argumentation hier auf die Bundesebene, doch hat die direktdemokratische Praxis in den Ländern nicht nur durch spektakuläre Entscheidungen von sich reden gemacht, sondern enthält auch politikwissenschaftlich äußerst relevante Lehren für die Folgen der Implementierung des direkten in das repräsentative Demokratieprinzip. ${ }^{32}$ Auf nationaler Ebene ist die Bundesrepublik dagegen noch ein weißer Fleck, was direkte Demokratie angeht, auch wenn diese seit längerem von der SPD, der FDP und den Grünen gefordert wird.

Bei der Frage nach der Einführung von Verfahren auf Bundesebene und ihrer möglichen Funktionsweise sind die institutionellen Besonderheiten des politischen Systems zu beachten. Dazu gehört neben dem deutschen Mehrebenensystem auch die Rolle des Bundesverfassungsgerichtes. Entgegen der direktdemokratischen Praxis in den Bundesländern plädiert Frank Decker dafür, auf der Bundesebene auf ein offenes Verfahren zu verzichten. Damit würde die Problematik der Einbeziehung des Bundesrates bei Initiativen, die den Föderalismus betreffen, automatisch wegfallen. Stattdessen kommen vier alternative direktdemokra-

30 Frank Decker, Die Systemverträglichkeit der direkten Demokratie, in: ZPol, 12. Jg. (2005), H. 4, S. $1103-1148$, S. 1105.

31 Vgl. stellvertretend Andreas Kost (Hrsg.), Direkte Demokratie in den deutschen Ländern. Eine Einführung, Wiesbaden 2005.

32 Hier sind besonders die Spannungen zwischen Volksentscheid und repräsentativen Akteuren in Hamburg zu nennen, denen an dieser Stelle allerdings nicht weiter nachgegangen werden kann. Vgl. Frank Decker, Parlamentarische Demokratie vs. Volksgesetzgebung. Der Streit um ein neues Wahlrecht in Hamburg, in: ZParl, 38. Jg. (2007), H. 1, S. $118-133$. 
tische Verfahren in Betracht: (1) eine konsultative Gesetzesinitiative, (2) die Vetoinitiati$\mathrm{ve}^{33}$, (3) das einfache Referendum und (4) das obligatorische Referendum. In diesen Fällen wäre die Systemverträglichkeit mit den in Deutschland entscheidenden Faktoren Primat des Grundgesetzes, föderative Mitregierung und Parteienwettbewerb vorhanden. ${ }^{34}$

Auf der Basis der hier vorgelegten Ergebnisse kann als erstes festgehalten werden, dass nicht der Fehler gemacht werden sollte, den Regierungsparteien die Möglichkeit einzuräumen, bei einfachen Gesetzen ein Referendum anzusetzen: Das dürfte wenig zur Reduzierung parteipolitischer Blockaden und zu sachorientierten Reformen beitragen, da diese Verfahren eher zu einem Instrument im parteitaktischen Arsenal der Parteien werden. Die Analyse hat auch gezeigt, dass offene Verfahren von Parteien eher sachorientiert eingesetzt werden. Doch ist schon darauf hingewiesen worden, dass sich die Implementierung einer Gesetzesinitiative in das politische System der Bundesrepublik aufgrund der Politikverflechtung nicht empfiehlt. Referendumsinitiativen dagegen haben den Nachteil, dass sie nicht konstruktiv wirken, sondern reine Veto-Instrumente sind, auch wenn sie eine andere Akteursarena eröffnen. So bleibt noch das obligatorische Referendum, das durch seine institutionelle Konstruktionsweise „politics“-orientierte Motive der Parteien nahezu ausgeschlossen hat. Wenn also ein Instrument außerhalb des deutschen Parteienstaates gefunden werden sollte, dann dieses.

Wie könnte ein solches Verfahren ins institutionelle System auf Bundesebene eingefügt werden, damit es eine entsprechende Wirkung als Instrument gegen parteipolitische Blockaden entfalten kann? Als entscheidend für die „policy“-orientierte Nutzung hat sich die Automatisierung der Kompetenz zur Auslösung erwiesen, die bei Referenden wiederum der Schlüssel zur parteitaktischen Nutzung gewesen ist. Die folgende Konstruktion würde diesen Implikationen Rechnung tragen. Dazu müsste das Verfahren von Verfassungsänderungen auf der Bundesebene wie es im Artikel 79 Absatz 2 des Grundgesetzes festgelegt ist wie folgt geändert werden: „Ein solches Gesetz bedarf entweder der Zustimmung von zwei Dritteln der Mitglieder des Bundestages und mindestens der absoluten Mehrheit der Stimmen des Bundesrates, oder der Zustimmung der absoluten Mehrheit der Mitglieder des Bundestages und zwei Drittel der Stimmen des Bundesrates, oder der Zustimmung der absoluten Mehrheit der Mitglieder des Bundestages und der Zustimmung der absoluten Mehrheit der Stimmen des Bundesrates sowie der Bestätigung durch die Mehrheit der Stimmen in einem obligatorischen Referendum. Im Falle einer Zustimmung durch die absolute Mehrheit der Mitglieder des Bundestages und einer Ablehnung durch die absolute Mehrheit aber von weniger als zwei Dritteln der Stimmen des Bundesrates, entscheidet obligatorisch die Mehrheit der Stimmen in einem Referendum. Im Falle einer Zustimmung von zwei Dritteln der Mitglieder des Bundestages und einer Ablehnung von zwei Dritteln der Stimmen des Bundesrates soll grundsätzlich ein Vermittlungsverfahren eingeleitet werden.“

Grundgesetzänderungen bedürften demnach nicht mehr zwangsläufig einer Zweidrittelmehrheit in beiden Häusern, wobei dennoch nicht vom Prinzip des höheren Bestandsschutzes der Verfassung abgewichen würde. Sie beginnen danach immer im Bundestag, wo sie entweder mit einfacher Mehrheit oder mit Zweidrittelmehrheit angenommen werden können. Die Höhe der Annahme signalisiert den parteipolitischen Konfliktgrad und ent-

33 Deckers Vetoinitiative entspricht hier der Referendumsinitiative.

34 Vgl. Frank Decker, a.a.O. (Fn. 30), S. 1140. 
scheidet damit auch über den weiteren Verlauf des Verfahrens. Es ergibt sich eine erste Variante mit einem nur geringen parteipolitischen Konfliktpotential und eine zweite mit umgekehrten Vorzeichen, bei der das obligatorische Referendum zum Tragen kommt, um eine parteipolitische Blockade zu verhindern.

In der ersten Variante ist die Annahme einer Verfassungsänderung im Bundestag mit einer Zweidrittelmehrheit erfolgt. Da die Regierungsfraktionen im Bundestag bislang nie über eine solche Mehrheit verfügten, kann von einem parteiübergreifenden Konsens ausgegangen werden, der die Logik von Regierung und Opposition außer Kraft setzt. Die Vorlage wird sodann dem Bundesrat zugeleitet. Nimmt dieser an, und zwar mindestens mit einfacher Mehrheit, ist das Grundgesetz geändert. Der besondere Bestandsschutz der Verfassung ist durch das einmalige Überspringen der Qualifizierungshürde gewahrt, und zugleich deutet die Annahme auf keinen signifikanten Konflikt mit Länderinteressen hin. Wird die Vorlage dagegen abgelehnt, bestimmt die Höhe der Ablehnung über das weitere Prozedere. Bei einer Zurückweisung durch bis zu zwei Drittel der Stimmen ist von gemeinsamen Länderinteressen auszugehen, die berührt wurden. Da der Konsens im Bundestag so groß war, ist es unwahrscheinlich, dass hinter der Bundesratsablehnung parteipolitische Motive stehen, denn es galt schon vorher, im Bundestag Farbe zu bekennen. Eine Zustimmung im Bundestag und eine Ablehnung im Bundesrat wäre unglaubwürdig und unwahrscheinlich. Dennoch wäre die Verfassungsänderung damit gescheitert. Liegt die Ablehnung allerdings noch über zwei Dritteln, deutet dies auf ein gravierendes Problem im Mehrebenensystem hin, so dass der Vermittlungsausschuss zum Einsatz käme. In dieser Variante spielt direkte Demokratie als „Exit“-Mechanismus keine Rolle, da durch die institutionelle Konstruktionsweise klar wird, dass es sich nicht primär um ein parteipolitisches Blockadespiel handelt.

Dies ist in der zweiten Variante, in der von einem parteipolitischen Konflikt mit entsprechender Blockade ausgegangen wird, anders. Hier erfolgt die Annahme einer Grundge-

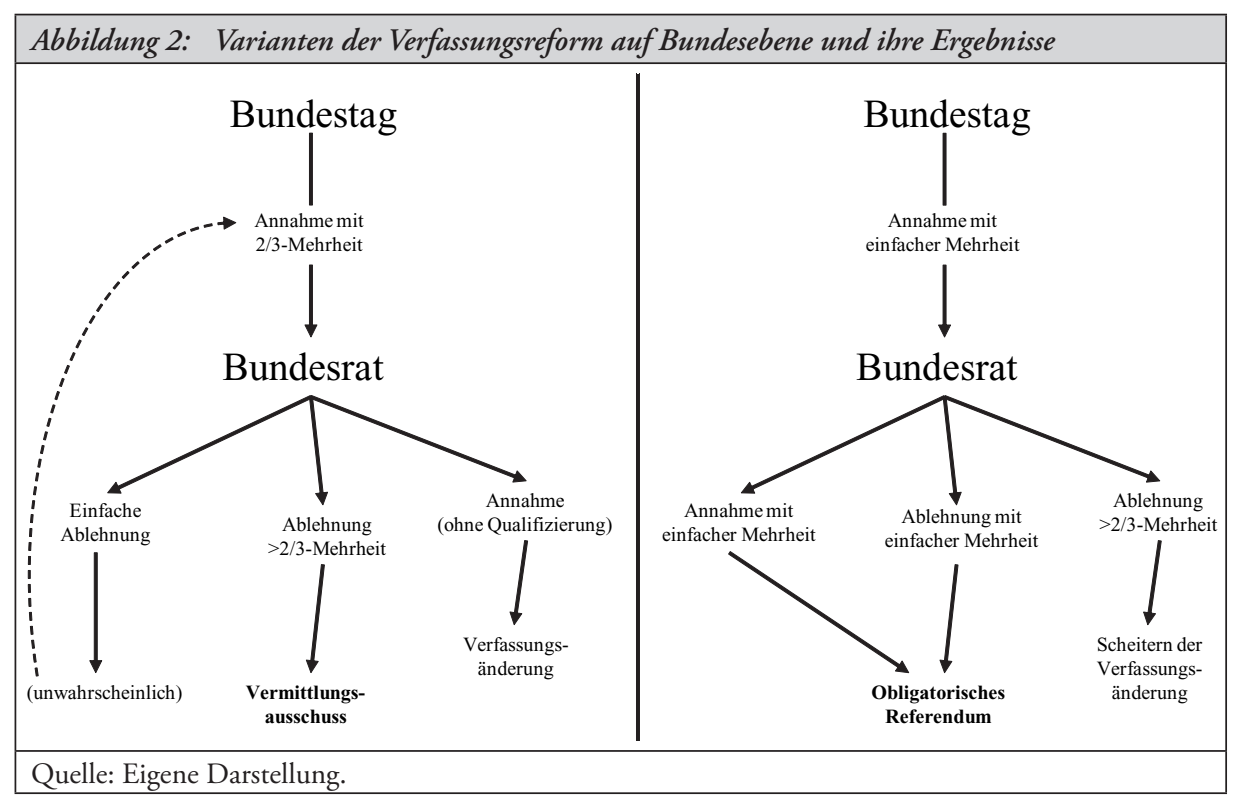


setzänderung im Bundestag mit der einfachen Mehrheit der Regierungsfraktionen. Klar ist hier der parteipolitische Konflikt um die Vorlage, wenn bedacht wird, dass Regierungsfraktionen in der Regel nur über eine absolute aber keine Zweidrittelmehrheit im Bundestag verfügen. Im Bundesrat ergeben sich wieder drei unterschiedliche Varianten, die vom dortigen Abstimmungsergebnis abhängen. Wird die Vorlage nur mit einfacher Mehrheit angenommen oder sogar abgelehnt, kommt es aus zwei Gründen zum obligatorischen Referendum. Erstens dient eine Abstimmung als zusätzliche Legitimationsinstanz für eine Grundgesetzänderung. Ansonsten würden einfache Mehrheiten in beiden Häusern ausreichen, was dem besonderen Bestandsschutz der Verfassung widerspricht. Der zweite Fall bezieht sich dagegen auf die parteipolitische Blockade, die bei einer recht knappen Ablehnung im Bundesrat zu vermuten ist, wenn beide Häuser parteipolitisch unterschiedlich dominiert sind. An diesem Punkt könnte eine Blockade nur um ihrer selbst Willen stattfinden. Die automatische Auslösung eines obligatorischen Referendums könnte so einem Stillstand begegnen und die - vermutlich parteipolitische - Ablehnung des Bundesrates überstimmen. Der Wähler könnte Reformprojekte der Regierungsparteien auch gegen den Willen der Opposition bestätigen. Nur wenn die Ablehnung im Bundesrat bei mehr als zwei Dritteln liegt, ist die Verfassungsänderung gescheitert, da hier vermutlich vitale Interessen zur gemeinsamen Position der Länder geführt haben. In diesem Fall überstimmen die Länder mit ihrer Zweidrittelmehrheit die einfache Annahme im Bundestag. Instrumentalisierungen der Bundesratsstimmen durch die Parteien erwiesen sich wieder dadurch schwierig, dass sie schon vorher im Bundestag Position beziehen mussten.

Diese Konstruktionsweise eines obligatorischen Referendums auf Bundesebene würde nur schwer parteitaktische Motive bei Regierung und Opposition ermöglichen. Die Mediation fällt auf beiden Seiten weg, da es bereits im Bundestag gilt, Farbe zu bekennen und parteiinterne Spaltungen keine Rolle spielen können. Auch die Agendafunktion als zweites parteitaktisches Motiv kommt für beide Seiten nicht infrage, da die Regierung nicht die Herbeiführung der Abstimmung beeinflussen kann. Die Opposition kann ihrerseits den Prozess der Verfassungsänderung aus ihrer Position gar nicht einleiten, um eventuell eine Abstimmung vorzuziehen. Die parteitaktische Legitimation (Legitimation 2) im Sinne einer Stärkung der eigenen Position im Parteienwettbewerb ist dagegen als Auslösungsmotiv unter diesen institutionellen Bedingungen zumindest bei den Regierungsparteien möglich. Sie könnten ein Verfassungsreformprojekt angehen, von dem sie wissen, dass es die Opposition schwächen würde. Bei den Oppositionsparteien kommt dieses Motiv dagegen nicht

\begin{tabular}{|l|c|c|}
\hline \multicolumn{2}{|c|}{ Tabelle 6: Motive beim obligatorischen Referendum getrennt nach Regierungs- und Oppositions- } \\
parteien
\end{tabular}


zum Tragen, da sie wiederum aus ihrer Position einen Reformprozess nicht einleiten können. Es bleiben also nur noch „policy“-orientierte Motive übrig. Auf der Seite der Regierungsparteien sind dies in erster Linie die legislative und die Legitimationsfunktion, während das Veto wegfällt. Auf der Seite der Oppositionsparteien ist dagegen das legislative Motiv obsolet, da sie ansonsten einfach zustimmen könnten. Naheliegend ist ein Veto. Vorstellbar ist aber auch das Legitimationsmotiv, wenn eine Entscheidung als so grundlegend erachtet wird, dass sie einer besonderen Bestätigung bedarf.

Es zeigt sich also, dass eine solche Konstruktion die Vorteile, die obligatorische Referenden vor dem Hintergrund der „politics“-,policy“-Dichotomie aufweisen, hervorbringen würde. Die Wahrscheinlichkeit einer Opposition um der Opposition willen kann zumindest reduziert und die Sachorientierung bei den Parteien erhöht werden. Andere zentrale Fragen zur Einführung direktdemokratischer Verfahren auf Bundesebene, die Frank Decker aufwirft ${ }^{35}$, müssten allerdings noch geklärt werden. Um dem Primat der Verfassung zu entsprechen, könnte zum Beispiel vor der Einleitung der Volksabstimmung in der Phase der Überleitung einer Vorlage vom Bundestag zum Bundesrat die Anrufung des Bundesverfassungsgerichts ermöglicht werden.

\section{Fazit und Ausblick: Das obligatorische Referendum - nicht zusätzlicher Input-, sondern Entscheidungskanal}

Die hier dargestellte Konstruktion eines direktdemokratischen Verfahrens im bundesdeutschen Föderalismus ist weder als Plädoyer für noch gegen die Einführung von Volksabstimmungen in repräsentative Systeme zu verstehen. Vielmehr sollte gezeigt werden, was aus der westeuropäischen direktdemokratischen Praxis für die Frage nach dem Reformbeitrag solcher Verfahren zu lernen ist. ${ }^{36}$ Der theoretische Ausgangspunkt war, dass Reformen am ehesten dort durchsetzbar sind, wo die Parteien nicht parteitaktisch agieren oder agieren können, also „policy“-Motive dominieren, während eine kurzfristige „politics“-Orientierung abträglich ist.

131 Abstimmungen in neun europäischen Ländern wurden daraufhin untersucht, welche Motive die Parteien dazu bewegen, direktdemokratische Verfahren anzuwenden. Danach haben die direktdemokratischen Prozesse viel Raum für langfristige, „policy“-orientierte Politik eröffnet. Am häufigsten sind obligatorische Referenden frei von parteitaktischem Kalkül gewesen, danach Initiativen und am wenigsten Referenden.

Die Einführung eines obligatorischen Referendums wird auch ansonsten in der Literatur als am ehesten kompatibel mit dem deutschen Institutionensystem betrachtet, so dass es gute Gründe gibt, auch für Deutschland eine Konstruktion zu entwerfen, die der ermittelten „policy“-Prägung anderer derartiger Verfahren in Westeuropa entspricht. Die hier vorgeschlagene Ausgestaltung eines obligatorischen Referendums würde die Wahrscheinlichkeiten von parteipolitisch motivierten Blockaden durch den Bundesrat auflösen, diesen

35 Vgl. ders., a.a.O. (Fn. 5), S. 7.

36 Vgl. stellvertretend für die immer wieder diskutierten Erwägungen zu Nutzen und Risiken direktdemokratischer Verfahren: Heidrun Abromeit, Nutzen und Risiken direktdemokratischer Instrumente, in: Claus Offe (Hrsg.), Demokratisierung der Demokratie. Diagnosen und Reformvorschläge, Frankfurt am Main 2003, S. 95 - 110. 
aber zugleich durch die verschiedenen Entscheidungsoptionen und Handlungsläufe nicht zugunsten direkter Demokratie entmachten. Zudem würden eher heikle Aspekte wie Quoren oder Themenausschlüsse durch die Verfahrenskonstruktion von vornherein vermieden. Klar sollte sein, dass ein obligatorisches Referendum kein zusätzlicher Input-Kanal sein, aber immerhin die vorhandenen Kanäle vielleicht freihalten kann. Wie die Bürger dann abstimmen werden, ist aber andere Frage.

\title{
Raucher oder Nichtraucher - ist das die Frage? Wahlbeteiligung und Abstimmungsverhalten beim Volksentscheid über das Rauchverbot in Bayern
}

\author{
Harald Schoen, Alexander Glantz und Rebecca Teusch
}

\section{Nichtraucherschutz in Bayern - eine unendliche Geschichte?}

Am 4. Juli 2010 waren knapp 9,4 Millionen Bayern dazu aufgerufen, in einem Volksentscheid über eine Verschärfung des Nichtraucherschutzes zu entscheiden. Damit fand eine längere Auseinandersetzung über das Rauchen im öffentlichen Raum, vor allem aber in Gaststätten, ihr - vorläufiges - Ende. ${ }^{1}$ Im Dezember 2007 beschloss der Bayerische Landtag das bis dahin bundesweit strikteste Rauchverbot. Das einzige Schlupfloch, das das Gesetz gelassen hatte, nutzten etliche Raucher und richteten bayernweit Raucherclubs ein. Nach der Landtagswahl 2008, bei der die CSU die absolute Mehrheit verloren hatte ${ }^{2}$, wurde das Rauchverbot gelockert. Die neue Landtagsmehrheit aus CSU und FDP führte verschiedene Ausnahmen beim Nichtraucherschutz ein, unter anderem für kleine Kneipen, Raucherräume und Festzelte. ${ }^{3}$ Auf diese Entscheidung hin initiierte die ÖDP mit Nichtraucherverbänden und Ärzten eine Unterschriftensammlung, um ein Volksbegehren „Für echten Nichtraucherschutz!“ zugunsten eines ausnahmslosen Rauchverbots in der Gastronomie einzuleiten. Dies gelang, und ebenso war das Volksbegehren erfolgreich, da vom 19. November bis zum 2. Dezember 2009 knapp 14 Prozent der Stimmberechtigten - und damit deutlich mehr als die erforderlichen zehn Prozent - eine Unterschrift zugunsten des Begehrens leisteten. Der Landtag lehnte daraufhin mit den Stimmen von CSU und FDP den Gesetzentwurf des Volksbegehrens ab und ebnete damit den Weg für einen Volksentscheid.

1 Vgl. zur Vorgeschichte des Volksbegehrens Bärbel Weixner, Nichtraucherschutz in Bayern - der Weg eines erfolgreichen Volksbegehrens und Volksentscheids im Freistaat, in: Lars P. Feld / Peter M. Huber / Otmar Jung / Christian Welzel / Fabian Wittreck (Hrsg.), Jahrbuch für direkte Demokratie 2010, Baden-Baden 2011, S. 255 - 294.

2 Vgl. dazu Rainer-Olaf Schultze / Jan Grasnick, Die bayerische Landtagswahl vom 28. September 2008: Betriebsunfall oder Ende eines Mythos?, in: ZParl, 40. Jg. (2009), H. 1, S. 34 - 55.

3 Vgl. Gesetz zum Schutz der Gesundheit (Gesundheitsschutzgesetz - GSG), Bayerisches Gesetzund Verordnungsblatt (GVBl), München 2009, S. 384. 